\title{
Lunar Theories
}

\section{A Heliocentric Suggestion That Calls for Some Attention}

\section{By J. Hardcastle}

SeEING recently (not for the first time) in Flammarion's "Popular Astronomy" his diagram representing the courses of the earth and moon around the sun as long-drawn-out wavy lines, crossing and recrossing each other, with his accompanying statement that the course of the moon is everywhere concave to the sun the humorous idea occurred to me that the motions of the earth and moon resembled those of two cyclist a]ternately "pacing" each other round an enormously long and narrow racing track. Such motions are entirely different from those usually ascribed to these bodies-the earth circling round the sun in an elliptical orbit, and the moon, so to speak, "running rings round" the earth in a smaller elliptical orbit. Struck by this difference of ideas, I set about inquiring whether the motions of the moon have been treated by anyone fror the heliocentric or any other extra-terrestrial point of view; looking upon earth and moon not as planet and satellite but as a pair of planets traveling round the sun in company, and changing places as leader and follower under the influence of their mutual gravitation
I have been able to find not even an illusion to such I have been able to find not even an illusion to such
treatment of the moon's motions. It is not because the true motion was not known. Thêre is Flammarion' diagram (first drawn in 1876 he tells us) and his ac companying remarks. These are explanatory of the diagram, not of the motions it represents. R. A. Proctor's "The Moon" (1st ed. 1873) also contains a diagram of a different kind, to illustrate his statement gram of a different kind, to illustrate his statement the earth, her track being a "serpentine or wavy line." I'roctor says the perturbations of the motions of the moon as a planet subject to the attractions of anothe planet- our own earth-could be easily tested. But h does not test them-does not treat the moon in that way. He insists that the sun always has nearly twice as much power over the moon as the earth has, yet he treats the earth as principal and the sun's attraction as a perturbing force merely; and throughout his minute treatise on the moon's motions he assumes that she travels in an elliptic orbit around the earth.

All the text-books, every encyclopaedia article and the like, that I have met with, explain the moon's motions as these are seen from the moving earth. In working out the moon's real motions as these would be seen from a viewpoint distant from the earth, found the problem of the apparent circular movement so simple and so interesting that I felt surprise that this treatment has not been adopted in "popular" as this treatment has not been adopted in "popular" as
tronomies, if only as a supplement to the usual geocentronomies, if only as a supplement to the usual geocen-
tric or "satellite" theory. The latter may be more con venient and more useful for the practical astronomerI cannot say. It may be easier to account for the minor motions-the perturbations or irregularities in the principal motions-by the geocentric theory than by the other. I cannot say. I suspect the contrary, because the geocentric theory deals with appearances,
and science, we are told, deals as far as possible with and science, we are told, deals as far as possible with
the realities behind appearances. As yet astronomy, as far as I can discover, has not attempted to to this in developing the theory of the moon's motions. It is stated that there are about sixty "anomalies" in the moon's motions, some of which have not yet been accounted for. A heliocentric lunar theory could scarcely involve more such enigmas. Proctor mentions one enigma that presents itself in the current theory, "If the sun has more power over the moon than the earth, why does it not pull the moon away from the earth at new moon?" Proctor's reply is a lame one, is no reply at all in fact, and it proves that he had not really thought of the moon as a planet subject to perturbations by the earth. Proctor does not deal with the principal motion of the moon, because "the phases of the moon are explained in text-books of astronomy."

"The earth goes round the sun in its annual journey of 365 days. The moon goes around the earth once every 27 days. The motion of the moon is thus a very complicated one, for it is actually moving round a body which is itself in constant motion." That is the explanation of the text-books, as stated by Sir Robert Ball, and he gives the moon's orbital rate as averaging 2,273 miles per hour. A very different notion is course in space.

The principal observable motions of the moon, as seen from the earth, are two. First, the familiar movement which is completed in a lunar month, or
lunation, which results in an apparent circuit of the earth, and in the production for us of the moon's "phases." It will be convenient to refer to this later as the "phase movement." Second, the movement in latitude, or northing and southing. Everyone has observed this, if only to the extent of noting that the ful moon is sometimes nearly overhead and sometimes low down in the north, at midnigh

For the principal movement, that which causes the moon to appear to travel round the earth, the facts or tance between earth and moon is about 60 times the tance between earth and moon is about 60 times the earth's radius or semi-diameter, or 238,000 miles. (2)
The earth's mass, gravitating or pulling power upon other heavenly bodies, is 80 times that of the moon, so that in a given time their mutual gravitation could move the moon towards the earth 80 times as far as it could move the earth towards the moon. (3) Consequently, from (1) and (2), the earth could pull the moon over the distance separating them in the time require by the moon to pull the earth over three-
fourth of the latter's radius. (4) The mean rate at fourths of the latter's radius. (4) The mean rate at
which earth and moon travel round the sun is 46 milwhich earth and moon travel round the sun is 46 mil-
lion miles per lunar month. (5) Earth and moon travel round the sun in a circular twin orbit, and are therefore subject to centrifugal force tending to take them away from the sun, this force therefore counteracting the sun's pull upon them towards itself. Acceleration of orbital rate increases, and lessening of that rate decreases, the centrifugal force. (6) The increases and decreases of orbital rate we have to deal with are but small fractions of that rate, in the case of the moon about one-fiftieth, in the case of the earth a quite minute fraction. (7) Both earth and moon describe wavy lines in their journey round the sun. A nearly pure circle is trace by the common centre of gravity of the two bodies, and that centre lies within the earth, on the line adjoining the centres of earth and moon, about three-fourths of the radius from the earth's centre when the moon is at its mean distance, and centre when the moon is at its mean distance, and
shifting 110 miles or so inward or outward with the moon's least and greatest distance, but always remaining within the earth. I will call the curve traced by that common centre of gravity the E.M. orbit. (8) In considering the phase movement in a general way the gravitational pull of the sun upon the earth and upon the moon may be assumed to be a constant quantity; and variations of the moon's distance from the earth, and the northing and southing of the moon may be igand the northing and southing of the moon may be ig-
nored. The daily rotation of the earth and the monthly rotation of the moon do not enter into the problem of the phases.

Starting with the moon at first quarter, with both earth and moon centred on the E.M. line (as sometimes happens) the moon is then following the earth at her mean distance, and both are, for the moment, travelling on the E.M. line at the same rate of $\mathbf{4 6}$ million miles per lunar month. In these positions the earth pulls the moon forward, increasing her solar orbit pace, and the moon forward, increasing her solar orbit pace, and the
moon pulls the earth backward, reducing its pace, the effective action of each upon the other being in proportion to their respective masses, i. e., 80 to 1 . These interactions continue for a week, in which time the moon has been drawn forward so that she overtakes the earth at full moon. The earth's gravitational ef fort has been to pull the moon to itself, but the increase in the moon's pace caused by this effort increases the moon's centrifugal force in the solar orbit, and this throws her further out from the sun, so that she passes the earth, at full, on the outside of the E.M. orbit. The acceleration and the centrifugal force are so nicely balanced that the moon maintains an approximately equal distance from the earth during the week, and she passes the earth almost as far away from it as she was at first quarter. The backward pull of the moon reduces the earth's orbital rate and its centhe moon reduces the earth's orbital rate and its cen-
trifugal force, so that the sun is enable to draw the earth inward, to the extent of three-fourths of the radius. (This follows from the fact that the common centre of gravity of the two bodies describes the common solar orbit.) Three-fourths of the earth's radius is also the measure of the amount the moon was able to retard the earth's progress in that week.

After the moon has passed the earth at full, and onward for a fortnight to new moon, opposite reactions take place. The earth pulls the moon back, reducing her rate and centrifugal force; the moon pulls the earth forward, increasing these elements in its case. The acceleration of the moon's rate between first quarter and full moon carries her ahead of the earth, but immediately she gets ahead the earth's pull becomes a brake, in a week deprives the moon of her acceleration, and reduces her centrifugal force so that the sun draws her inward to the E.M. line at last quarter. At this point the moon is still ahead of the earth, and the brake action continues to slow down the moon so that the earth passes her at new moon. The sun meanwhile, acting upon the moon's lessened centrifugal force, has drawn her inward to the moon-earth distance inside the E.M. line at new. During the period from full to new moon the earth is drawn forward, its orbit rate is slightly increased, and consequently at new moon the centre of the earth is outside the E.M. orbit by threefourths of the radius.

After new moon their mutual gravitation accelerates the moon's and lessens the earth's rate, consequently the moon swings out to the E.M. line at first quarter, our starting point, and the earth swings back to centre on that line. The alternate accelerations and retardations of her
solar orbit rate cause the moon, in passing from first to last quarter, to travel nearly half a million miles (twice the earth-moon distance) further than in the other half of the lunation; for, starting at first quarter that distance behind the earth, she is at last quarter the same distance ahead of the earth; and these gains are lost through her slower pace between last and first quarters. During each lunation the earth alternately gains and loses pace, at times opposite to the gain and loss of the moon, to an extent of twice three-fourths of her radius, or about 12,000 miles.

It must constantly be born in mind that these changes in pace, resulting in changes of relative position, are but small fractions of the enormous orbit rate of 46 million miles per lunation; and that each increase and It must is spread, though unequally, over a fortight. by the moon in swinging outward from the sun differ very little in curvature from the E.M. orbit, because they are about a hundred times as long as the extreme width separating them from that orbit. If we draw part of a circle with a radius of 92 inches (it can be done with a bit of twine and a pencil) and cut off a segment of it with a line about 59 inches long we shall have an arc of about 30 degrees, and 60 inches long, representing a month's course of the E.M. centre of gravity. If we would add the moon's course along this arc in due proportion, we must draw the two curves, outer and inner, so that their extreme distances, at full and new, from the E.M. line, are but little more than one-third of an inch from that line. It will be seen at once that the moon's course is always concave towards the sun, and consequently that centrifu
always acting effectively upon the moon.

For a time I wondered, and others may wonder also, if the moon has to travel faster to overtake the earth, and then to slow down to allow the earth to overtake her, why these differences of rate are not clearly perceptible in the apparent movements of the moon. As one friend said, "The moon has to go 'eyes out' to over take the earth, and we ought to see that." Such wonder arises from forgetting that the overtaking of the earth is spread over a week, and that the increase of distance travelled is but a small fraction of the total journey in that period. As a matter of fact the swifter and slower paces of the moon are observed, measured and calculated in advance, and given in the almanacs under the heading "Right Ascension at Noon," for each succeeding day. Comparison of the differences between successive pairs of these numbers shows where the accelerations and retardations occur.

To sum up this part of the subject: It appears to me that the monthly changes in the moon's position in reation to the earth and sun-the phase-making movement-are explained by referring them to alternate increases and decreases in the centrifugal force in her solar orbit, due to the attraction of the earth alternately increasing and decreasing her solar orbit rate, the opposing forces being so well balanced that the moon maintains an almost constant distance from th earth. (The difference on either side of the mean distance is at most one-fourteenth.) The result is that, as seen from the earth, the moon appears to travel round the earth only.

Reverting to the cyclists "pacing" motion, it is now clear that this will not do. Luna does some pacing, and Tellus feebly responds to the invitation to "come on," but when Luna has dropped back to the rear to
give Tellus his turn, he also slows down. Tellus in fact, maintains an almost even pace in the middle o 
the track. Luna races up, passes on the right, gets ahead a little, then slows down, and, observing the rule of the road, allows Tellus to pass her, and she falls back as far to the rear as she had previously been ahead. That it is not a case of Luna "running rings round" Tellus will be seen from the fact that the proportions of their monthly course may be likened to a cycle track, slightly curved, two miles long, and, to give plenty of room, $120 \mathrm{ft}$. wide, on which two cyclists travel together. One of them, the bigger, Tellus, has a start of $60 \mathrm{ft}$., and rides in the middle of the track all the way. The other, Luna, overtakes Tellus at the half mile, and having swung out to the right-hand side half mile, and having swung out to the right-hand side
of the track passes him. At the end of a mile Luna is $60 \mathrm{ft}$. ahead of Tellus, and in the middle of the track. In the second mile Luna slackens her pace, swings across to the inner side of the track, Tellus passes her represents the month's course, Luna is again $60 \mathrm{ft}$. represents the month's course, Luna is again $60 \mathrm{ft}$.
behind Tellus. This is the true order of their going, behind Tellus. This is the true order of their going,
month after month, as far as concerns the apparent month after month, as far as concerns the apparent
circling round the earth and the phenomena of the circling round the earth and the phenomena of the
phases. The "elliptical orbit" is but the special terrestrial view of that order. An eye placed high above the plane of the earth's orbit would see it very differently, and as here set forth. It follows, therefore, that the Man in the Moon could frame a theory of the motions of the earth, as seen from the moon, on all fours with our current lunar theory. He could say that the earth moved round the moon in an elliptical orbit, having the moon in one of the foci of the ellipse. That would be his explanation of the earth's phases and differences in the apparent size of the earth, and other conditions would be given associated explanations.

The phase movement of the moon, considered separately from other movements, might be confined to the ecliptic or plane of the earth's orbit. In that case, to an observer on the sun the fortnightly excursions of the observer on the sun the fortnightly excursions of the
moon inside and outside the E.M. orbit would not be moon inside and outside the E.M. orbit would not be
discernible, except by change of apparent size with change of distance. The moon would appear to travel on the same track as the earth, alternately leading and
following, alternately passing backward in front of the earth, and forward on the further side.

The second of the moon's chief motions, her northing and southing, or swing in latitude, similarly considered as if it alone existed, is visible to a supposed observer as if it alone existed, is visible to a supposed observer
on the sun, as a wavy line, running in a very flat curve on the sun, as a wavy line, running in a very flat curve
for a fortnight above (north of) the earth's track, and then in an equally flat curve for a fortnight below (south of) it. These curves are shorter than the phase curves, as 12 to $13-421 / 2$ million miles for each pair, as against 46 millions. The width of the curves is very much less. The phase curves run out to 238,000 miles as a mean and 253,000 at the maximum, while the latitude curves at most run out to about 22,000 miles, or
less than one-eleventh of the other's maximum, and less less than one-eleventh of the other's maximum, and less
than three times the earth's diameter. It takes the moon 29.53 days to run a pair of phase curves; a pair of latitude curves are traced in 27.212 days, say 2.3 days less.

This swing in latitude is a true "swing," a pendulum like movement. Starting slowly from each extreme, north or south, it increases in pace till the ecliptic is crossed, slows down to the other limit and then slowly crossed, slows down to the other limit and then slowly
starts back again. The almanacs, in their tables of starts back again. The almanacs, in their tables of
"declination of the moon at noon," foretell the position of the moon in latitude for each day, and comparisons
of the daily changes show that this is the nature of the of the daily
movement.

The geocentric theory explains that this swing in latitude is a consequence of the moon's orbit being "inclined to the ecliptic," by a fraction over five degrees. The "declinations," however, usually reach over grees. The "declinations," however, usually reach over
28 degrees. This is because to the inclination of the 28 degrees. This is because to the inclination of the
moon's orbit is added the inclination of the earth's axis to the ecliptic, $231 \frac{1 / 2}{2}$ degrees. The moon's "tropics" on the earth are therefore 57 degrees apart, and at some part of each lunation the moon reaches further north or south on the meridian than does the sun at midsummer, and descends lower than does the sun at midwinshare in the total declination is only 5 degrees on each share in the total declination is only 5 degrees on each
side of the ecliptic, or working in the fraction over, side of the ecliptic, or working in the fraction over,
about 22,000 miles north and south of the ecliptic. In a diagram representing the earth by a circle $5 \% \frac{3}{4}$ inches in diameter, with axis and equator drawn at an angle of $231 / 2$ degrees to a horizontal line drawn through the centre representing the ecliptic, one line above and one below the ecliptic line, parallel with and a quarter of an inch from it, would

Both sets of curves, phase and swing, being traced Both sets of curves, phase and swing, being traced
by one body, they must next be conceive in their combination. As a complete oscillation to and fro in latitude takes less time than a lunation or phase series (as
27.2 days is to 29.5 days), and therefore extends over a shorter length of orbit (as $421 / 2$ million miles is to 46 millions) the crests of successive latitude curves occupy different positions among the phase curves. The swing sometimes carries one phase, sometimes another, to its extreme north and south of the ecliptic. When the southern swing in latitude coincides with the extreme outer movement of the phases our full moon is high on the meridian at midnight. When the southern swing coincides with the extreme inward movement our new moon appears far south of west, having travelled during the day high in the sky, and the next full moon will be low down in the north. The north or south swing may be completed when, in the absence of this motion, the moon would be on the E.M. line, that is, at a quarter, and in these cases that quarter will be high in our sky, and the next, preceding and following, will be low. A series of such changes is gone through in a year, therefore they are seasonal, though not precisely true to calendar dates, the difference being about the same as with the dates of the "moveable
feast" of Easter. Proctor points out that the fact of these positions of the moon being seasonal ought to put an end to notions that the moon has any special influence on the weather

It is quite easy to mentally visualise the phase movement and the swing in latitude movement separately, but the combination of the two sets of curves, at right angles to each other, of different lengths, and of very different curvature, results in a curious much flattened
spiral around the E.M. line that requires much patience to follow through successive months. The spiral is so flattened that its thickness, so to speak, is less than one-eleventh of its width

Astronomers in their investigations of the complicated motions of the moon take note of several "periods" and relate "orbits." One orbit is defined by its production of the phases, another by its inclination to the ecliptic, which results in the northing and southing, a third is the orbit which is elliptic and has the sun in one of its foci. All these orbits have differen "periods." The elliptic orbit is taken to be the prin cipal orbit, probably because astronomers first began with calculations of the moon's varying distances, and other orbits are worked upon as the results of perturbations. An interesting fact comes out on considering the differences among the periods. As seen from a the differences among the periods. As seen from a
distant star the moon completes a rotation and a revodistant star the moon completes a rotation and a revo-
lution in a "sidereal" month of 27.322 days; a series of phases is completed in a "synodical" month of 29.53 days; the orbit which is inclined to the ecliptic is traversed in a "nodical" month of 27.212 days; the orbit which is the ellipse with the earth in one of its foci is traversed in an "anomalistic" month of 27.55 days. There is also a "tropical" month, a small fraction shorter than the sidereal month. A little simple arithmetic shows that thirteen sidereal periods give an (approximate) year of 355.16 days; thirteen nodical months a year of 355.16 days, thirteen anomalistic months a year of 358.16 days; and twelve synodical months a year of 354.36 days. These (approximate) lunar years are all shorter than our solar and civil year of $3651 / 4$ days. The point I wish to draw attention to is this. If earth and moon travelled in a straight line instead of a circular one, other conditions of their journey remaining the same, there would be thirteen phase months in the year instead of twelve, and the phase movements would keep step with the swing movements, if not absolutely, then very nearly. Earth and moon, however travel in a circular course, not a straight line, and also slowly gyrate about their common centre of gravity, like a pair of waltzers going round a room. The consequence in both cases is to the performers' loss of count of one gyration. The dancers would count one count of one gyration. The dancers would count one
turn less than a spectator in the balcony would see. The law that a revolution around a centre (whether the course be a circle or of any other form) is equivalent to a rotation about its own centre, has many familiar applications. A garden hose, for example, is twisted in coiling it, and one must get rid of that twist by twisting the hose in the opposite way. Binder twine is a very slackly spun cord, and the twist in it is visibly tightened if it is wound round a small thing in one tightened if it is wound round a small thing in one
direction, and visibly loosened if wound in the opposite direction. In the case of the earth and moon the loss of a sidereal month is spread throughout the year, and results in each phase month being lengthened by onetwelfth of the missing month, and twelve phase months nodical months. This loss of count from within of one revolution counted from outside the system affects the earth and the moon individually. Seen from without, the earth makes 3661/4 rotations in our year of $3651 / 4$ days.

As this movement in latitude, considere as an inde- pull of the sun, so that this pull cannot be its cause: and as the principal action of the earth produces the much larger phase movements, the swing in latitude must, I think, be due to some special influence exerted by the earth. The regular periodicity of the swing implies a like periodicity in its cause, that is, the force must be applied at intervals as regular as those of the swing. A familiar illustration of this is the child's swing. One pushes it, not continuously, but at intervals, and to be satisfactory the pushes must have intervals, and to be satisfactory the pushes must have
the same intervals as the swings. As the motion of the moon in latitude is of the nature of a swing and the moon, balanced between the pulls of the sun and the earth on the one hand and her centrifugal force on the other is free to be moved, a very small force, regularly applied, would suffice to start and maintain pull of the moon on the nearer and further halves of the earth's "equatorial bulge" has an important effect upon the earth. I cannot work it out; but it seems probable that the moon's swing of 5 degrees in latitude is caused by the reaction of the earth's equatorial bulge - her retaliation, for the moon's plucking at her girdle. The diagram above specified can be usefully applied in this connection, in order to see how the nearer half of the bulge in certain positions must pull upon the moon at a different angle from the earth as a whole, moon at a different angle from the earth as a whole,
which is considered to exert its force as if this were which is considered to exert its force as if this were
concentrated at its centre. Of course any part of the earth's gravitating force applied to produce the swing in latitude must by so much reduce the total that, in the absence of the swing, would be devoted to the suggested is at mid-summer and mid-winter most effective at new and full moon; at the equinoxes (spring and autumn) at the quarters; and mid-way between solstace and equinox at the octants of the moon. The latter positions would not be as favorable as the first two, but they provide twice as many opportunities for the pull of the earth's girdle. 'That the swing in latitude is due to the earth only, and to a cause that acts
regularly with each true (sidereal) revolution, is seen in the fact that there are thirteen to and fro oscillations in latitude in the lunar year. The cause suggested must act on the whole at right angles to the plane of must act on the whole at right angles to the plane of
the earth's axis (which is always parallel with itself) and at right angles to the ecliptic; hence its effects conform in number with the sidereal not with the solar, revolutions, and are not involved in the loss of count that affects the phase series.

Other points might be touche upon, but these are secondary, and this essay is not an attempt to produce a complete theory but merely an attempt to show that the motions of the moon can be studied from an extraterrestrial viewpoint with interesting results. Whether this can be done usefully or not for astronomical science it is for astronomers to say. There seems to be a confession of need for a new method of attacking the problem in some remarks by Professor Simon Newcomb, a well-known American astronomer, author of the article "Astronomy," in the "Encyclopaedia Britannica." Professor Newcomb says :- "The mathematical theory of the moon's motions does not yet form a well-defined body of reasoning and octrine, like other branches of mathematical science, but consists of a series of researches extending through twenty centuries or more, and not easily welded into a unified whole Although the precision of the mathematical solution has been placed beyond serious doubt, the problem of motiotely reconciling this solution with the observe Professor Newcomb's words, "beyond serious doubt," Professor Newcomb's words, "beyond serious doubt,"
suggest that he, who had devoted much time to lunar problems, had some loubt about the soundness of the current theory. At all events, if no one has yet attempted to develop a heliocentric theory the incompleteness hands giving the former a trial.
hand geontric theory is a good

\section{Gas for Raising Steam}

(Continued from page 179)

temperature of nearly $500 \mathrm{deg}$. In fact, the flame temperature, under such conditions, is so near to the ignition temperature of carbon monoxide that great diffculty might be experience in burning the gas even with the smallest excess of air. Thus it appears fairly evident that sensible heat must be sacrificed, and with a high flame of the calorific power, in of ignition.

$$
\text { EXCESS AIR. }
$$

The most serious losses occur through the admission to the boiler of any air in excess of the mintmum amount required for the combustion of the gas, the
excess quantity reducing the flame temperature and 